\title{
Collective dynamics of bursty particle precipitation initiating in the inner and outer plasma sheet
}

\author{
V. M. Uritsky ${ }^{1}$, E. Donovan ${ }^{1}$, A. J. Klimas ${ }^{2}$, and E. Spanswick ${ }^{1}$ \\ ${ }^{1}$ Physics and Astronomy Department, University of Calgary, Calgary, AB, Canada \\ ${ }^{2} \mathrm{UMBC}$ at NASA/Goddard Space Flight Center, Greenbelt, MD, USA
}

Received: 21 October 2008 - Revised: 8 January 2009 - Accepted: 8 January 2009 - Published: 16 February 2009

\begin{abstract}
Using multiscale spatiotemporal analysis of bursty precipitation events in the nighttime aurora as seen by the POLAR UVI instrument, we report a set of new statistical signatures of high- and low-latitude auroral activity, signaling a strongly non-uniform distribution of dissipation mechanism in the plasma sheet. We show that small-scale electron emission events that initiate in the equatorward portion of the nighttime auroral oval (scaling mode $A_{1}$ ) have systematically steeper power-law slopes of energy, power, area, and lifetime probability distributions compared to the events that initiate at higher latitudes (mode $B$ ). The low-latitude group of events also contain a small but energetically important subpopulation of substorm-scale disturbances (mode $A_{2}$ ) described by anomalously low distribution exponents characteristic of barely stable thermodynamic systems that are prone to large-scale sporadic reorganization. The high latitude events (mode $B$ ) can be accurately described by a single set of distributions exponents over the entire range of studied scales, with the exponent values consistent with globally stable self-organized critical (SOC) behavior. The lowand high latitude events have distinct inter-trigger time statistics, and are characterized by significantly different MLT distributions. Based on these results we conjecture that the inner and outer portions of the plasma sheet are associated with two (or more) mechanisms of collective dynamics that may represent an interplay between current disruption and magnetic reconnection scenarios of bursty energy conversion in the magnetotail.
\end{abstract}

Keywords. Ionosphere (Particle precipitation) - Magnetospheric physics (Storms and substorms) - Space plasma physics (Nonlinear phenomena)

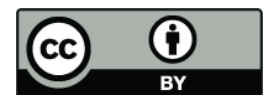

Correspondence to: V. M. Uritsky (vuritsky@phas.ucalgary.ca)

\section{Introduction}

Soon after the development of basic substorm phenomenology it was realized that the nighttime auroral oval is not a simple latitudinally bound distribution of emission brightness and electric currents. In fact, the activity of this part of the ionosphere is extremely complicated, and it incorporates a variety of effects reflecting different conditions in the coupled solar wind - magnetosphere - ionosphere system. Examples of these are substorm expansion onsets, pseudobreakups, steady magnetospheric convection events, bursty bulk flows, sawtooth events, and other processes (e.g. Zesta et al., 2000; Lui, 2001; Frey et al., 2004; Henderson et al., 2006).

It has also been found that despite the diversity of physical phenomena involved in the magnetospheric response to the changing solar wind driver, the output energy dissipation flux as estimated from particle precipitation in the nighttime aurora tends to cluster in intermittent spatiotemporal bursts described by robust scale-free statistics (Lui et al., 2000; Lui, 2002; Uritsky et al., 2003, 2002, 2006).

The term "scale-free" was coined in the statistical mechanics of turbulent and/or critical phenomena to describe correlated perturbations with no characteristic scales other than the scales dictated by the finite size of the system, as opposed to scale-dependent perturbations reflecting physical conditions that vary across different scales (Dhar, 2006; Sreenivasan et al., 2004; Lubeck, 2004). The observational signatures of the scale-free behavior include the power-law shape of probability distributions, Fourier spectra, autocorrelation functions, and other statistics, with the power-law exponents being constant over wide ranges of scales. Some well-known manifestations of such behavior in the geo- and space sciences are fully developed turbulence in hydrodynamic and magnetized flows (Lazarian, 2006), GuttenbergRichter statistics of earthquake magnitudes (Turcotte, 1989),

Published by Copernicus Publications on behalf of the European Geosciences Union. 
statistical and topological scale-invariance of flaring activity in the solar corona (Charbonneau et al., 2001).

Nighttime auroral activity provides an impressive example of scale-free behavior. The energy probability distribution of electron precipitation regions as seen by the POLAR satellite exhibits power-law shape over about 6 orders of magnitude (Uritsky et al., 2002). By combining POLAR data with ground-based TV observations (Kozelov et al., 2004), the power-law scaling range of energy distributions has been extended up to 11 orders of magnitude. However, these scale-free statistics represent long-term averaged properties of nighttime magnetospheric disturbance, and they can mask more complicated dynamics at the level of specific plasma structures responsible for the generation of various forms of auroral precipitation. Exploring these phenomena could help to build a more solid theoretical link between the statistical and dynamical plasma descriptions, to evaluate predictability of different magnetospheric states, and to obtain statistical guidelines for designing future space missions targeted at multiscale plasma disturbances.

In a companion paper (Uritsky et al., 2008), we demonstrated that inner and outer portions of the magnetotail plasma sheet are characterized by substantially different scaling regimes of bursty energy dissipation suggesting different kinds of plasma turbulence in these regions. This analysis was based on a simple subdivision of onset locations into groups of "high" and "low" latitude events relative to the line $66^{\circ}$ MLAT, which is given by the maximum in the distribution in latitude of these events.

Here we investigate in depth the scale-free and scaledependent modes of auroral precipitation dynamics using a more accurate classification method based on an empirical auroral oval model capturing nonlinear mapping effects, and applying a more comprehensive set of statistical tools. We compute an extensive collection of scaling exponents supplemented by quantitative measures of both random and systematic scaling errors as explained in Sect. 2.2. Our main new results include (1) demonstration of a qualitative agreement between the locations of the precipitation events detected using our spatiotemporal algorithm and the substorm onset positions in the database by Frey et al. (2004); (2) statistical proof of a separate group of strong low-latitude events described by a distinct set of scaling laws; (3) power-law exponents of cumulative distributions of emission event parameters found to be consistent with probability density exponents; (4) analysis of power-law distributions of the inter-trigger time of the events revealing distinct scaling exponents for the high- and low-latitude auroral regions; (5) comparison of the obtained distribution exponents with structure function exponents of the electric field fluctuations reported earlier.

These new results confirm the causal relationship between the auroral precipitation statistics and the underlying nonuniform morphology of the central plasma sheet (CPS). They show that the inner and the outer CPS regions are responsible for three distinct scaling modes of the auroral precipi- tation dynamics. Based on this picture, we propose a preliminary physical interpretation for the observed latitudinal dependence of the collective properties of nighttime precipitation events in terms of the current disruption and midtail reconnection scenarios of the CPS reconfiguration.

\section{Data and methods}

\subsection{Detection of spatiotemporal events}

Our analysis is based on a collection of digital images of nighttime northern aurora (55-80 MLAT, 20:00-04:00 MLT) obtained from the Ultraviolet Imager (UVI) onboard the POLAR spacecraft, in the 165.5 to $174.5 \mathrm{~nm}$ portion of the Lyman-Birge-Hopfield spectral band, using an integration time of $36.5 \mathrm{~s}$, and a time resolution of $184 \mathrm{~s}$. The studied database includes 16000 images taken during two observation periods (1 January 1997-28 February 1997 and 1 January 1998-28 February 1998), both close to a solar minimum. The images were rebinned down to a uniform spatial resolution of $70 \times 70 \mathrm{~km}$ which was kept constant irrespective of the spacecraft altitude.

In contrast to statistical approaches dealing with individual auroral images (Lui et al., 2000; Kozelov and Rypdal, 2007), our study involved spatiotemporal tracking of emission events as briefly explained below. In most cases, this tracking made it possible to identify the precipitation events that co-evolved simultaneously in multiple auroral locations and could not be resolved otherwise (Uritsky et al., 2002).

The UV luminosity $w(t, \mathbf{r})$ was analyzed as a function of time $t$ and position $\mathbf{r}$ on the image plane. First, active auroral regions were identified by applying the lower activity threshold $w_{a}$ representing a background UV flux. Adjacent spatial regions with $w(\mathbf{r}, t)>w_{a}$ were treated as parts of evolving events. By checking for overlap of common pixels between each pair of consecutive UVI frames, we identified a set of 3-dimensional spatiotemporal integration domains $\Lambda_{i}(i=1, . ., N)$ corresponding to each of the $N$ individual emission events found by this method. Thus, the events were defined as connected regions in space-time. These domains of contiguous activity were used to compute the lifetime, $T_{i}=\max \left(t \in \Lambda_{i}\right)-\min \left(t \in \Lambda_{i}\right)$, the energy, $\quad E_{i}=k \int_{\Lambda_{i}} w(\mathbf{r}, t) d \mathbf{r} d t$, the peak power, $W_{i}=k \max \left(\int_{\Lambda_{i}(t)} w(\mathbf{r}, t) d \mathbf{r}\right), \quad$ and the peak area, $A_{i}=\max \left(\int_{\Lambda_{i}(t)} d \mathbf{r}\right) \quad$ of every event. The factor $k=2.74 \times 10^{-8} \mathrm{~J}_{\text {photon }^{-1}}$ is an empirical constant for converting photon fluxes to energy fluxes (Brittnacher et al., 1997). In addition, we have computed the delay (inter-trigger) time $D$, defined as the time interval between the beginning of a given event and the beginning of the most recent preceding event.

The robustness of the obtained statistics was verified by repeatedly running the algorithm with substantially different $w_{a}$. Below we show the results for the threshold 
$w_{a}=10$ photons $\mathrm{cm}^{-2} \mathrm{~s}^{-1}$ for consistency with our previous publications (Uritsky et al., 2002, 2006).

\subsection{Statistical tools}

The initial position of each auroral event was estimated with a typical error of about $300 \mathrm{~km}$ in either spatial directions. This error is due to the fact that the starting area of the active auroral regions tracked by our algorithm was usually above the image resolution. The latitudinal positions $\phi_{i}$ were studied relative to the line given by an empirical model for the equatorward boundary of the auroral oval due to Gvozdevsky and Sergeev (1995):

$\phi_{i}=\operatorname{MLAT}_{i}-\left[67.9-4.3 \cos \left(\pi\left(\mathrm{MLT}_{i}-23.1\right) / 12\right)\right]$

Here, $\mathrm{MLT}_{i}$ and $\mathrm{MLAT}_{i}$ are the geomagnetic coordinates of the $i$-th event in our database, and the numerical constants represent average solar wind - magnetosphere coupling conditions (see Gvozdevsky and Sergeev, 1995, for details). The model was originally designed as a proxy for the isotropic boundary separating the inner magnetospheric region of adiabatic particle motion from the outer region of stochastic particle motion in the CPS.

In a strongly perturbed magnetotail state such as the one observed during to the expansion phase onset, the equatorward boundary model used in Eq. (1) fails to predict the correct isotropic boundary, which can move a considerable distance due to the tail stretching and dipolarization (Donovan et al., 2003; Meurant et al., 2007). However, this model remains a valuable tool for separating the inner (near-Earth) and the tail CPS regions in a typical magnetotail configuration.

The statistics of the emission events that initiated on the poleward $(\phi>0)$ and equatorward $(\phi<0)$ side of the auroral oval were characterized by sets of probability density distributions $p(x)$ and cumulative probability distributions $P(x)=\int_{x}^{\infty} p\left(x^{\prime}\right) d x^{\prime}$, where $x \in\{E, W, A, T, D\}$. The absolute values of the power-law exponents of the two groups of statistics are denoted correspondingly as $\tau_{x}$ and $\theta_{x}$, with the subscript indicating the variable under study. In every case, the validity of power-law approximations $\left(p(x) \propto x^{-\tau_{x}}, P(x) \propto x^{-\theta_{x}}\right)$ has been verified based on the standard regression errors as well as systematic scaling errors $\Delta_{x}=\tau_{x}-\theta_{x}-1$. The latter approach 0 in the scale-free case since

$$
\begin{gathered}
p(x) \propto x^{-\tau_{x}} \Rightarrow P(x) \propto \int_{x}^{\infty}\left(x^{\prime}\right)^{-\tau_{x}} d x^{\prime} \\
\propto x^{-\tau_{x}+1} \stackrel{\text { def }}{=} x^{-\theta_{x}} \Rightarrow \Delta_{x}=0 .
\end{gathered}
$$

We used logarithmic binning for computing both $p(x)$ and $P(x)$ distributions. The data points were merged into groups with logarithmically scaled boundaries providing a fixed number of bins per decade, which reduced the noise in the tails of the distributions resulting from limited number of

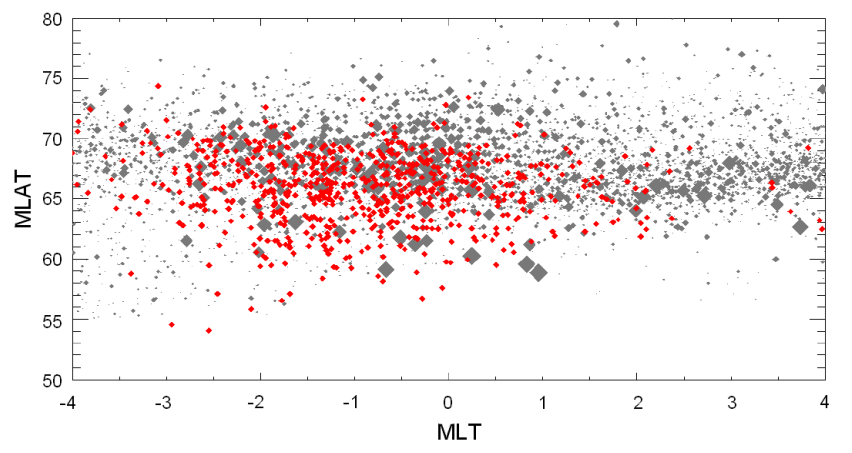

Fig. 1. Onset positions of the auroral emission events obtained using the spatiotemporal detection method described in the text (grey color, symbol size proportional to event energy $E$ ) superposed with the auroral substorm onsets (red) from the database by Frey et al. (2004).

large events. The accuracy of this approach is comparable with the methods based on maximum likelihood estimators, and is the best among graphical methods of power-law exponent evaluation (Bauke, 2007).

\section{Results}

\subsection{Data overview}

Figure 1 shows the onset positions of all the events detected by our spatiotemporal method $(n=7481)$. These positions are combined with the database of substorm onset positions constructed by Frey et al. (2004) using IMAGE FUV data. It should be understood that our database includes any auroral brightnings, which are not necessarily related to substorm activity. Nevertheless, the statistical overlap between the two data sets is reasonable, indicating that the auroral region prone to producing substorm breakups is also active in a broader sense.

The scatterplot in Fig. 2 represents the statistical dependence between the energy $E$ and the relative latitude $\phi$ of the events in our database. The shape of the scatterplot to the left and to the right of the $\phi=0$ line is notably different. The subset of high-latitude events (positive $\phi$ ) forms a uniform cloud of data points, with maximum energies observed at typical latitudinal positions of substorm breakups (Fig. 1). This energy peak extends towards negative $\phi$ events. However, the latter exhibit a wide gap of missing intermediate-energy activity, which suggests two distinct subpopulations in this $\phi$ range.

We have found that the asymmetry seen in Fig. 2 has important implications for the scaling properties of the activity in low- and high-latitude auroral regions. In our further analysis, we denote the small-scale and the large-scale emission events initiated at $\phi<0$ as mode $A_{1}$ and mode $A_{2}$ events, 


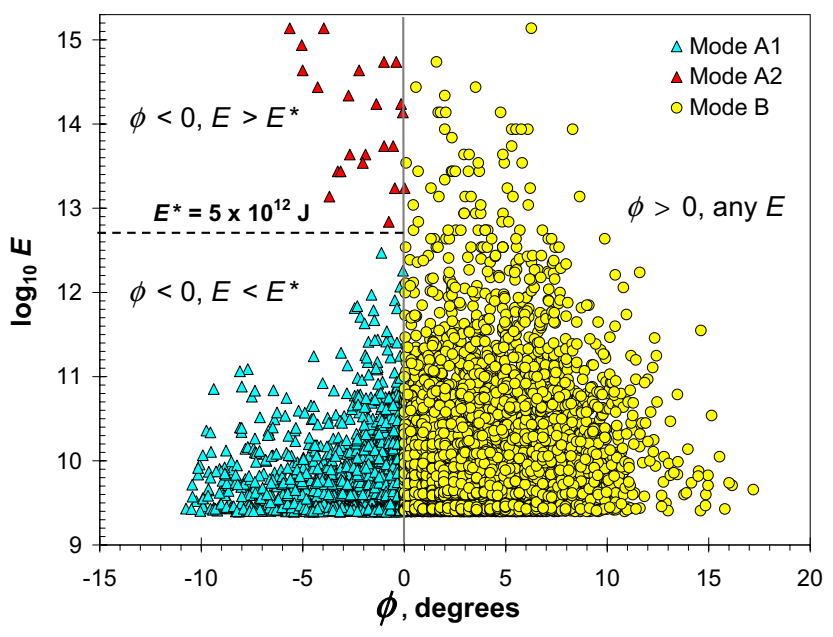

Fig. 2. Scatterplot of emission energies versus relative magnetic latitudes of onset locations for the three modes of emission dynamics described in the text. The dashed horizontal line marks the crossover energy separating mode $A_{1}$ and mode $A_{2}$ events.

respectively. The uniform $\phi>0$ population will be referred to as mode $B$ events.

\subsection{Event classification}

Figures 3-6 show probability distributions for the emission events which were initiated above $(\phi>0)$ and below $(\phi<0)$ the auroral boundary defined by Eq. (1). The numerical values of the scaling exponents describing the shape of these distributions are summarized in Table 1.

The results obtained indicate that $A_{1}, A_{2}$ and $B$ events have significantly different statistical features as discussed below.

High-latitude events: $\phi>0$. The events whose onsets are described by positive $\phi$ are characterized by broad-band power-law statistics with no distinct characteristic scales (scaling mode $B$ according to our classification). The scalefree nature of these events is confirmed by small systematic errors $\Delta_{x}$ (see Table 1) indicating a consistency of $\tau_{x}$ and $\theta_{x}$ exponents with the scaling relations (2) predicted for powerlaw distributions with single log-log slopes.

We note that the range of scales of this power-law behavior involves both small auroral activations and rather large events whose energy and power outputs lie in the range of fully developed substorms (Carbary et al., 2000). The exponents $\tau_{E}, \tau_{W}, \tau_{A}$ and $\tau_{T}$ are close to the corresponding values reported earlier for the same observation period without filtering the activity by the onset location (Uritsky et al., 2002, 2003, 2006).

Low-latitude events: $\quad \phi<0$. The events described by negative $\phi$ have a more complicated statistical pattern. Their probability functions demonstrate a crossover behavior
Table 1. Comparative parameters of the three scaling modes of the emission dynamics.

\begin{tabular}{lccc}
\hline Parameter & $A_{1}$ & $A_{2}$ & $B$ \\
\hline \multicolumn{3}{c}{ Defining characteristics } \\
Onset latitude & $\phi<0$ & $\phi<0$ & $\phi>0$ \\
$E, J$ & $<5 \times 10^{12}$ & $>5 \times 10^{12}$ & $10^{9}-10^{15}$ \\
& \multicolumn{3}{c}{ Relative contribution } \\
$\%$ of events & $19.3 \%$ & $\sim 0.3 \%$ & $80.4 \%$ \\
$E_{\Sigma}, J$ & $3.06 \times 10^{13}$ & $6.89 \times 10^{15}$ & $4.97 \times 10^{15}$ \\
$E_{\Sigma} \times 100 \%$ & $\sim 0.3 \%$ & $57.9 \%$ & $41.8 \%$
\end{tabular}

Scaling exponents \pm standard errors

\begin{tabular}{lccc}
$\tau_{E}$ & $1.93 \pm 0.04$ & $0.97 \pm 0.14$ & $1.63 \pm 0.02$ \\
$\theta_{E}$ & $0.92 \pm 0.02$ & $0.54 \pm 0.07$ & $0.62 \pm 0.01$ \\
$\Delta_{E}$ & $0.01 \pm 0.04$ & $-0.57 \pm 0.14$ & $0.01 \pm 0.02$ \\
& & & \\
$\tau_{W}$ & $2.45 \pm 0.20$ & $0.91 \pm 0.37$ & $1.88 \pm 0.02$ \\
$\theta_{W}$ & $1.11 \pm 0.05$ & $0.64 \pm 0.23$ & $0.93 \pm 0.01$ \\
$\Delta_{W}$ & $0.34 \pm 0.20$ & $-0.73 \pm 0.37$ & $-0.05 \pm 0.02$ \\
& & & \\
$\tau_{T}$ & $3.23 \pm 0.08$ & $-\mathrm{a}$ & $2.47 \pm 0.08$ \\
$\theta_{T}$ & $2.22 \pm 0.12$ & - & $1.51 \pm 0.05$ \\
$\Delta_{T}$ & $0.01 \pm 0.12$ & - & $-0.04 \pm 0.08$ \\
& & & \\
$\tau_{A}$ & $2.49 \pm 0.10$ & $0.88 \pm 0.26$ & $1.97 \pm 0.02$ \\
$\theta_{A}$ & $1.31 \pm 0.04$ & $0.76 \pm 0.18$ & $1.02 \pm 0.02$ \\
$\Delta_{A}$ & $0.18 \pm 0.10$ & $-0.88 \pm 0.26$ & $-0.05 \pm 0.02$ \\
& & & \\
$\tau_{D}$ & $1.72 \pm 0.05$ & $-\mathrm{b}$ & $2.05 \pm 0.11$ \\
$\theta_{D}$ & $0.86 \pm 0.03$ & - & $1.10 \pm 0.03$ \\
$\Delta_{D}$ & $-0.14 \pm 0.05$ & - & $-0.05 \pm 0.08$ \\
& & & \\
\hline
\end{tabular}

a,b Exponents omitted due to insufficient accuracy.

which includes small scale portions with $\tau_{x}$ and $\theta_{x}$ exponents considerably greater than the corresponding exponents of the high-latitude events, as well as large-scale portions exhibiting the opposite tendency. To quantify these crossovers, we divided the whole set of events with $\phi<0$ into two subgroups based on the criteria $E>E^{*}$ and $E<E^{*}$, where $E^{*}=5 \times 10^{12} \mathrm{~J}$ is the approximate energy level marking the transition between the steep and shallow log-log slopes in the $p(E)$ distribution. The exponents below and above $E^{*}$ have different values. Also, for small-scale events (scaling mode $A_{1}$ ), the relation $\tau_{x}-\theta_{x}-1=0$ approximately holds $\left(\Delta_{x}\right.$ errors are relatively small), while for large-scale events (scaling mode $A_{2}$ ), this relation is effectively invalid. These observations strongly suggest that the studied activity is, in fact, a scaledependent process whose dynamical features at small and 

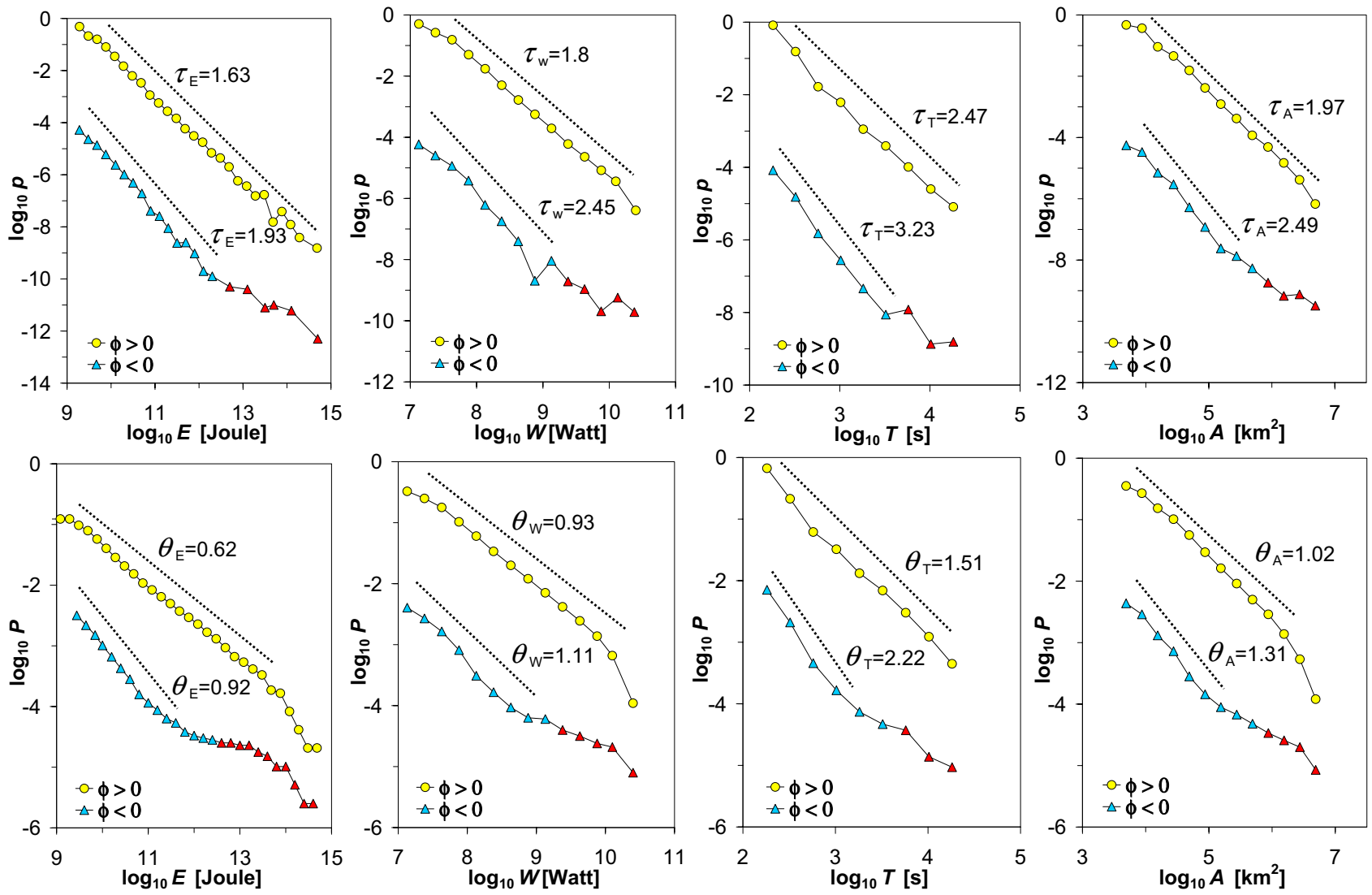

Fig. 3. Probability density (top row) and cumulative (bottom row) distributions of emission energy $E$, peak emission power $W$, emission lifetime $T$, and peak emission area $A$, constructed for precipitation events which initiated above and below the $\phi=0$ boundary. Low-latitude distributions are shifted downward for easier comparison. The plots reveal three distinct modes of ionospheric unloading described by significantly different distribution slopes: small-scale ( $A_{1}$ mode) and large-scale (mode $\left.A_{2}\right)$ events started at $\phi<0$, as well as the events which started at $\phi>0$ irrespective of their size ( $B$ mode). The dotted lines show linear regression slopes for $A_{1}$ and $B$ emission modes. Note the absence of distribution crossovers for the high-latitude events.

large emission scales are governed by substantially different physical mechanisms.

The existence of two separate subgroups of $\phi<0$ events is more evident in terms of the dependent variable $y=f(E) \equiv \log _{10}(E)$. Since $p(E)$ is a monotonic function, the $p(y)$ distribution is given by the probabilistic transform

$p(y)=\frac{p\left(f^{-1}(y)\right)}{f^{\prime}\left(f^{-1}(y)\right)}$

in which $f^{-1}=10^{y}$ is the inverse function and $f^{\prime}=\log _{10}(e) / E$ is the derivative of $f(E)$. Denote the power-law slopes of $p(E)$ below and above the threshold $E^{*}$ as $\tau_{E}^{(1)}$, and $\tau_{E}^{(2)}$, respectively. Then Eq. (3) yields

$p(y) \propto \begin{cases}10^{y\left(1-\tau_{E}^{(1)}\right)}, & y<\log _{10}\left(E^{*}\right) \\ 10^{y\left(1-\tau_{E}^{(2)}\right)}, & y \geq \log _{10}\left(E^{*}\right)\end{cases}$

For the negative $\phi$ population $\tau_{E}^{(1)} \approx 2$ and $\tau_{E}^{(2)} \approx 1$ (see Table 1), and so we expect the semilogarithmic plot $\log _{10}[p(y)]$ vs. $y$ to have a slope of -1 for $y<\log _{10}\left(E^{*}\right) \approx 12.7$ and to be horizontal otherwise. For the positive $\phi$ population (mode $B$ events), a single $\log _{10}[p(y)]$ slope of about -0.6 is expected. Figure 4 shows the actual data that confirm the predicted scaling behaviors. The distinction between the statistics of $A_{1}$ and $A_{2}$ is very clear, despite the small size of the $A_{2}$ population. It can also be seen that the boundary between the two scaling modes is fairly close to the selected energy threshold.

The inter-trigger time distributions (Fig. 5) of both highand low-latitude events have power-law form. The difference between the $\tau_{D}$ exponents of the low- and high-latitude populations is statistically significant $(p<0.05)$.

To complete the statistical picture, Fig. 6 shows the occurrence probability of precipitation events as a function of magnetic latitude and MLT. The energy threshold used for constructing these distributions is an order of magnitude lower that the one used in Figs. 3 and 4, which allowed us to include both large- and medium- size events associated with 


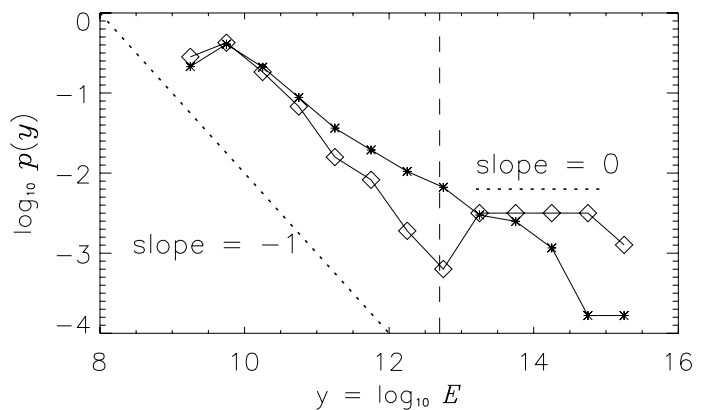

Fig. 4. Distributions of low-latitude (diamonds) and highlatitude (stars) emission events over the quantity $y \equiv \log _{10} E$. The pronounced break in the low-latitude distribution occurring at $E^{*}=5 \times 10^{12} \mathrm{~J}$ (shown with dashed vertical line) confirms the existence of two distinct subpopulations $\left(A_{1}\right.$ and $\left.A_{2}\right)$ in this auroral region. The slopes to the left and to the right of this break are consistent with the shape of the energy distribution $p(E)$ as discussed in the text.

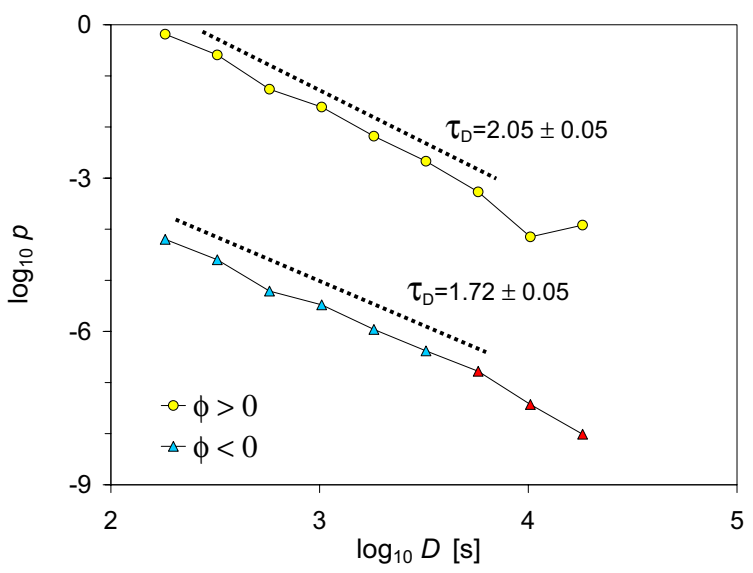

Fig. 5. Inter-trigger time distributions of low- and high-latitude events exhibiting distinct power-law slopes.

substorms and pseudobreakup activities. The MLAT distribution (built without pre-filtering by $\phi$ ) displays a single peak consistent with the average location of the electron aurora (Hartz and Brice, 1967; Frey et al., 2004). The MLT distributions for the negative and positive $\phi$ populations differ in their shape. The high-latitude events peak approximately an hour before magnetic midnight while the lower latitude events peak on the dawn side approximately $2 \mathrm{~h}$ after local midnight. This observation is consistent with our event classification as we discuss in the next section.
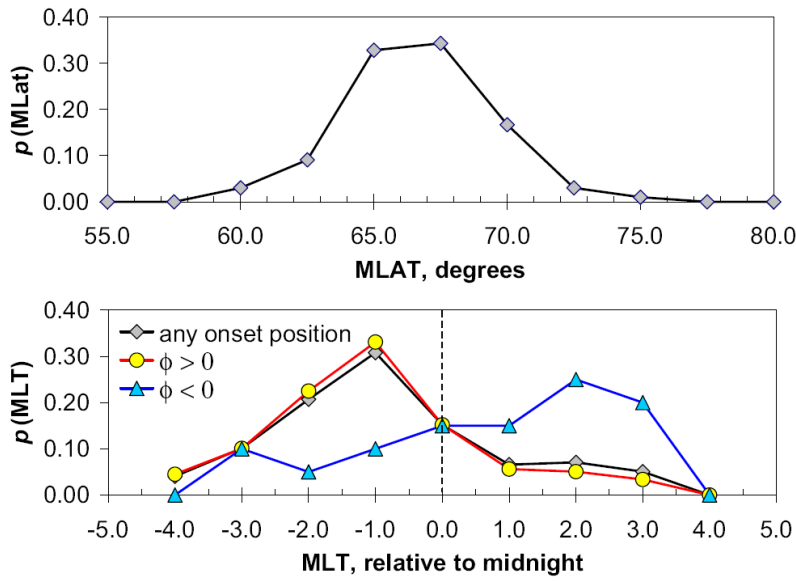

Fig. 6. Normalized occurrence frequency of magnetic latitudes (top) and magnetic local times (bottom) of medium- and large-scale events with $E>5 \times 10^{11}$. Note that the MLT distribution of the lowlatitude events peaks in the morning auroral sector.

\section{Discussion}

Our main findings can be briefly summarized as follows:

1. The emission events whose onsets are located poleward of the average isotropic boundary are characterized by broad-band power-law statistics with no distinct characteristic scales;

2. The events that initiate equatorward of this boundary constitute a non-uniform statistical population with an energy crossover separating large and small auroral activations;

3. The scaling behavior of the low- and high-latitude activity is different within the entire range of scales studied.

Statistically, mode $A_{1}$ events have a relatively high occurrence frequency but a vanishing precipitation energy (see Table 1). The strong low-latitude events ascribed to the scaling mode $A_{2}$ are quite infrequent. However, due to their large size, they carry almost $60 \%$ of the precipitated energy and thus represent a very important aspect of the auroral dissipation.

Mode $B$ appears to be the most common scaling mode responsible for as much as $4 / 5$ of the total number of the observed emission events. This explains why the scale-free behavior of high-latitude events is close to the overall auroral statistics reported in our previous publications Uritsky et al. (2002, 2003, 2006). The energy output of the mode $B$ events is also very significant (more than $40 \%$ of the entire auroral emission energy deposition), which implies that the scalefree magnetotail activity responsible for this emission mode plays a considerable, if not dominating, part in the intermittent bursty activity of the nightside magnetosphere. 
The power-law shape of the $p(D)$ distributions (Fig. 5) may indicate that the triggering mechanism that starts the emission events involves a highly correlated driver, such as e.g. the reconnection rate at the dayside magnetopause, the global cross-tail current, or the magnetic pressure in the tail lobes, which introduces a long-range coherence of the dissipation bursts beyond the one reflected by their lifetime distributions. If this interpretation is correct, our results imply that this driver has a more organized dynamics in the case of small-scale low-latitude events $\left(\tau_{T}-\tau_{D}=1.51\right.$, mode $A_{1}$ ) than it does in the case of high-latitude events $\left(\tau_{T}-\tau_{D}=0.42\right)$. However, it should be noted that our definition of $D$ is different from the definitions of "waiting" or "quiet" times in avalanching systems that have been studied in this context earlier (Sanches et al., 2002; Paczuski et al., 2005). This difference leaves a possibility that the observed long-range correlations of the onset timing originate from an intrinsic CPS dynamics rather than from an external driving process.

Our analysis suggests a consistent picture in which the mode $B$ events initiate in the outer CPS regions, with the strongest emission bursts occurring in a rather stretched magnetic field configuration. The energy conversion in this region is believed to be dominated by magnetic reconnection. To the extent the electron emission flux can be used as a proxy for the magnetic energy release in the tail (Shue et al., 2003), our results show that the midtail reconnection is a highly turbulent bursty process with no well-defined dissipation scales. Its scale-free component has been succcessfully reproduced in a driven current sheet simulation (Klimas et al., 2004). Interestingly, this simulation yielded a set of critical exponents which are fairly close to the exponents characterizing mode $B$ emission events in our present analysis. Judging by its relative energy contribution (Table 1), the scale-free dynamics in the reconnection regions has a considerable impact on the nightside magnetosphere.

The mode $A_{1}$ and $A_{2}$ events defined by negative $\phi$ values are mainly produced in the inner CPS. Due to a more stable magnetic field topology, this region is not a preferred location for the magnetic reconnection. However, it can be prone to current disruption, which offers an alternative mechanism for the energy release in the inner tail. The anomalously low distribution exponents observed for $A_{2}$ events are characteristic of barely stable thermodynamic systems that are prone to large-scale sporadic reorganization. This type of statistics may be a manifestation of an avalanching behavior of localized dipolarization regions in the near-Earth CPS region (Lui, 2002; Consolini et al., 2005).

The differing MLT peaks seen in Fig. 6 which reflect a large-scale inhomogeneity of plasma sheet provide additional evidence for the magnetotail origin of the observed effects. The two populations seen in this figure have previously been identified in auroral data (see Hartz and Brice, 1967, and references therein) and can be explained by the access of plasma sheet electrons to the inner magnetosphere. Elec- trons are naturally driven to the dawnside magnetosphere due to gradient curvature drifts, with the radial location of the population controlled, in large part, by the competing crosstail and co-rotation electric fields. For any given energy and species of inner CPS particle the path that it takes through the system is completely controlled by these factors (e.g. Friedel et al., 2001). On average, the transport of electrons through the system will bring them closer to Earth on the dawn-side than in the evening sector, in agreement with our observations.

Our results characterize the collective behavior of the emission events in the nightside sector of the auroral oval. Several recent publications focused on the electric field fluctuations in the same region (Abel et al., 2006, 2007; Parkinson, 2006, 2008). Although the scaling exponents reported in these papers describe a different aspect of auroral complexity, they can be reconciled with our analysis assuming that the electric field and the emission intensity fluctuations are generated by the same physical phenomenon. If the auroral turbulence is driven by an avalanching process in the tail, one can expect that the ratio $z$ between the spatial $(\alpha)$ and the temporal $(\beta)$ exponents of the second-order structure function of the electric field fluctuations obeys the theoretical scaling relation $z=\alpha / \beta=\gamma(2-\tau)$ (Paczuski et al., 1996; Aegerter et al., 2003), where $\tau \equiv \tau_{E}$ and $\gamma$ is the finite-size scaling exponent of the $p(E)$ distribution.

Based on our present results, it is clear that $z$ should exhibit a strong latitudinal dependence. Indeed, substituting the $\tau_{E}$ values from Table 1 , and using the previously obtained average value $\gamma=2.14$ characterizing the electron aurora (Uritsky et al., 2006), we find that $z=0.15,2.35$, and 0.79 for the scaling modes $A_{1}, A_{2}$, and $B$, respectively. The spatial and temporal electric field scaling exponents reported by Abel et al. (2006) and Parkinson (2006) for the auroral region equatorward of the open-closed boundary relate to each other as $0.39 / 0.21 \approx 1.86$. In the monofractal approximation (Parkinson, 2008), this ratio should be equal to $z$, and the scaling relation given above should hold. Using the estimates of $z$ given above, one can conjecture that the correlation structure of the auroral electric field fluctuations is largely controlled by the $A_{2}$ mode of bursty CPS dynamics, which is consistent with the dominating energy contribution of this mode.

In order to prove this link in a more rigorous way, a more careful analysis will be needed in which all of the exponents involved in the discussed relation will be measured for the same set of auroral events organized by latitude according to the same procedure. It is worth noting that the relationship between the structure function and avalanche exponents is at the heart of the ongoing search for a unified complexity scenario reconciling intermittent turbulence and SOC (Uritsky et al., 2007; Rypdal et al., 2008). The proposed analysis could provide an experimental basis for validating such a scenario in the magnetosphere.

It should be emphasized that the magnetosphereionosphere interaction may play a noticeable role in the 
observed effects. Although it is unlikely that the distinct scaling regimes reported here are created by this interaction, it could well lead to additional systematic errors of measured scaling exponents (for example, by filtering-out small-scale magnetotail activity, or by introducing more complicated distortions caused by plasma turbulence in the acceleration region), which will require careful consideration in future studies.

\section{Conclusions}

We have reported a new set of statistical signatures of electron emission events in the northern aurora based on an automated spatiotemporal analysis of POLAR UVI images. Our analysis has revealed several distinct types of precipitation dynamics defined by the initial location of the events relative to the average position of the isotropic boundary. The events that initiate in the equatorward portion of the auroral oval exhibit bifractal energy, power, area, and lifetime probability distributions described by different sets of power-law exponents referring to small and large emission scales. The small-energy events (mode $A_{1}, E<5 \times 10^{12} \mathrm{~J}$ ) are described by the distribution slopes that are systematically steeper than the corresponding slopes of large-energy events (mode $A_{2}$, $E>5 \times 10^{12} \mathrm{~J}$ ). The events that initiate in the poleward portion of the auroral oval (mode $B$ ) demonstrate robust scalefree monofractal behavior described by constant distribution slopes over the entire range of the observed precipitation energies.

The probability density exponents shown in Table 1 are approximately consistent with $\tau_{x}$ exponents obtained earlier from a less accurate latitudinal classification of emission events (Uritsky et al., 2008). The new exponents $\theta_{x}$ and $\Delta_{x}$ confirm the scale-dependent nature of the low-latitude emission dynamics, whereas the exponent $\tau_{D}$ suggests more organized driving and/or dissipation mechanisms underlying the activity in the inner CPS region.

The emission events produced in the outer CPS have considerably higher relative occurrence compared to the inner CPS events and therefore dominate the overall auroral statistics, making the scale-dependent behavior of the equatorward portion of the auroral oval a second-order effect in the statistical sense. However, the contribution of the equatorward events to the energy budget of the nighttime aurora is important and it needs to be addressed in future models of multiscale dissipation in the magnetotail.

A natural next step in this research line is to look for patterns, within our database, of event classes we know to exist. For example, what subset of our event database would be classified as substorms? Do substorms organize themselves in this manner in the parameter space of auroral scaling exponents? And if so, can this analysis be used as an independent test for alternative substorm onset scenarios?
Acknowledgements. We thank W. Liu for stimulating discussions, G. Parks and D. Chua for POLAR UVI data, A. Richmond for APEX coordinate conversion algorithms. This work was supported in part by the Canadian Space Agency.

Topical Editor R. Nakamura thanks M. L. Parkinson and $\mathrm{K}$. Rypdal for their help in evaluating this paper.

\section{References}

Abel, G. A., Freeman, M. P., and Chisham, G.: Spatial structure of ionospheric convection velocities in regions of open and closed magnetic field topology, Geophys. Res. Lett., 33, L24103, doi: 10.1029/2006GL027919, 2006.

Abel, G. A., Freeman, M. P., Chisham, G., and Watkins, N. W.: Investigating turbulent structure of ionospheric plasma velocity using the Halley SuperDARN radar, Nonlin. Processes Geophys., 14, 799-809, 2007, http://www.nonlin-processes-geophys.net/14/799/2007/.

Aegerter, C. M., Gunther, R., and Wijngaarden, R. J.: Avalanche dynamics, surface roughening, and self-organized criticality: Experiments on a three-dimensional pile of rice, Phys. Rev. E, 67, 2003.

Bauke, H.: Parameter estimation for power-law distributions by maximum likelihood methods, European Phys. J. B, 58, 167173, 2007.

Brittnacher, M., Elsen, R., Parks, G., Chen, L., Germany, G., and Spann, J.: A dayside auroral energy deposition case study using the POLAR Ultraviolet Imager, Geophys. Res. Lett., 24, 991994, 1997.

Carbary, J. F., Liou, K., Lui, A. T. Y., Newell, P. T., and Meng, C. I.: "Blob" analysis of auroral substorm dynamics, J. Geophys. Res., 105, 16083-16091, 2000.

Charbonneau, P., McIntosh, S. W., Liu, H. L., and Bogdan, T. J.: Avalanche models for solar flares, Solar Phys., 203, 321-353, 2001.

Consolini, G., Kretzschmar, M., Lui, A. T. Y., Zimbardo, G., and Macek, W. M.: On the magnetic field fluctuations during magnetospheric tail current disruption: A statistical approach, J. Geophys. Res., 110, A07202, doi:10.1029/2004JA010947, 2005.

Dhar, D.: Theoretical studies of self-organized criticality, Physica A, 369, 29-70, 2006.

Donovan, E. F., Jackel, B. J., Voronkov, I., Sotirelis, T., Creutzberg, F., and Nicholson, N. A.: Ground-based optical determination of the b2i boundary: A basis for an optical MT-index, J. Geophys. Res., 108, 1115, doi:10.1029/2001JA00919, 2003.

Frey, H. U., Mende, S. B., Angelopoulos, V., and Donovan, E. F.: Substorm onset observations by IMAGE-FUV, J. Geophys. Res., 109, A10304, doi:10.1029/2004JA010607, 2004.

Friedel, R. H. W., Henderson, M. G., Thomsen, M. F., and Scudder, J. D.: Plasma sheet access to the inner magnetosphere, J. Geophys. Res., 106, 5845-5858, 2001.

Gvozdevsky, B. B. and Sergeev, V. A.: MT-index - A possible new index to characterize the configuration of the magnetotail, ThreeDimensional Magnetosphere, 18, 51-54, 1995.

Hartz, T. R. and Brice, N. M.: The general pattern of auroral particle precipitation, Planet. Space Sci., 15, 301-329, 1967.

Henderson, M. G., Skoug, R., Donovan, E., Thomsen, M. F., Reeves, G. D., Denton, M. H., Singer, H. J., McPherron, R. L., Mende, S. B., Immel, T. J., Sigwarth, J. B., and Frank, L. A.: 
Substorms during the 10-11 August 2000 sawtooth event, J. Geophys. Res., 111, A06206, doi:10.1029/2005JA011366, 2006.

Klimas, A. J., Uritsky, V. M., Vassiliadis, D., and Baker, D. N.: Reconnection and scale-free avalanching in a driven currentsheet model, J. Geophys. Res., 109, A02218, doi:10.1029/ 2003JA010036, 2004.

Kozelov, B. V. and Rypdal, K.: Spatial scaling of optical fluctuations during substorm-time aurora, Ann. Geophys., 25, 915-927, 2007, http://www.ann-geophys.net/25/915/2007/.

Kozelov, B. V., Uritsky, V. M., and Klimas, A. J.: Power law probability distributions of multiscale auroral dynamics from groundbased TV observations, Geophys. Res. Lett., 31, L20804, doi: 10.1029/2004GL020962, 2004.

Lazarian, A.: Intermittency of magnetohydrodynamic turbulence: An astrophysical perspective, Int. J. Modern Phys. D, 15, 1099 1111, 2006.

Lubeck, S.: Universal scaling behavior of non-equilibrium phase transitions, Int. J. Modern Phys. B, 18, 3977-4118, 2004.

Lui, A. T. Y.: Current controversies in magnetospheric physics, Rev. Geophys., 39, 535-563, 2001.

Lui, A. T. Y.: Multiscale phenomena in the near-Earth magnetosphere, J. Atmos. Solar-Terr. Phys., 64, 125-143, 2002.

Lui, A. T. Y., Chapman, S. C., Liou, K., et al.: Is the dynamic magnetosphere an avalanching system?, Geophys. Res. Lett., 27, 911-914, 2000.

Meurant, M., Gerard, J. C., Blockx, C., Spanswick, E., Donovan, E. F., Hubert, B., Coumans, V., and Connors, M.: EL - a possible indicator to monitor the magnetic field stretching at global scale during substorm expansive phase: Statistical study, J. Geophys. Res., 112, A05222, doi:10.1029/2006JA012126, 2007.

Paczuski, M., Maslov, S., and Bak, P.: Avalanche dynamics in evolution, growth, and depinning models, Phys. Rev. E, 53, 414443, 1996.

Paczuski, M., Boettcher, S., and Baiesi, M.: Interoccurrence times in the Bak-Tang-Wiesenfeld sandpile model: A comparison with the observed statistics of solar flares, Phys. Rev. Lett., 95, 181102-1-181102-4, 2005.

Parkinson, M. L.: Dynamical critical scaling of electric field fluctuations in the greater cusp and magnetotail implied by HF radar observations of F-region Doppler velocity, Ann. Geophys., 24, 689-705, 2006, http://www.ann-geophys.net/24/689/2006/.
Parkinson, M. L.: Complexity in the scaling of velocity fluctuations in the high-latitude F-region ionosphere, Ann. Geophys., 26, 2657-2672, 2008, http://www.ann-geophys.net/26/2657/2008/.

Rypdal, K., Kozelov, B., Ratynskaia, S., Klumov, B., Knapek, C., and Rypdal, M.: Scale-free vortex cascade emerging from random forcing in a strongly coupled system, New J. Phys., 10, 093018, doi:10.1088/1367-2630/10/9/093018, 2008.

Sanches, R., Newman, D. E., and Carreras, B. A.: Waiting-time statistics of self-organized-criticality systems, Phys. Rev. Lett., 88, 068302-1-068302-4, 2002.

Shue, J. H., Ohtani, S., Newell, P. T., Liou, K., Meng, C. I., Ieda, A., and Mukai, T.: Quantitative relationships between plasma sheet fast flows and nightside auroral power, J. Geophys. Res., 108, 1345, doi:10.1029/2002JA009794, 2003.

Sreenivasan, K. R., Bershadskii, A., and Niemela, J. J.: Multiscale SOC in turbulent convection, Physica A, 340, 574-579, 2004.

Turcotte, D. L.: Fractals in geology and geophysics, Pure Appl. Geophys., 131, 171-196, 1989.

Uritsky, V. M., Klimas, A. J., Vassiliadis, D., Chua, D., and Parks, G.: Scale-free statistics of spatiotemporal auroral emissions as depicted by POLAR UVI images: Dynamic magnetosphere is an avalanching system, J. Geophys. Res., 107, 1426, doi:10.1029/ 2001JA000281, 2002.

Uritsky, V. M., Klimas, A. J., and Vassiliadis, D.: Evaluation of spreading critical exponents from the spatiotemporal evolution of emission regions in the nighttime aurora, Geophys. Res. Lett., 30, 1813, doi:10.1029/2002GL016556, 2003.

Uritsky, V. M., Klimas, A. J., and Vassiliadis, D.: Critical finitesize scaling of energy and lifetime probability distributions of auroral emissions, Geophys. Res. Lett., 33, L08102, doi:10.1029/ 2005GL025330, 2006.

Uritsky, V. M., Paczuski, M., Davila, J. M., and Jones, S. I.: Coexistence of self-organized criticality and intermittent turbulence in the solar corona, Phys. Rev. Lett., 99, 1669-1676, 2007.

Uritsky, V. M., Donovan, E., Klimas, A. J., and Spanswick, E.: Scale-Free and Scale-Dependent Modes of Energy Release Dynamics in the Nighttime Magnetosphere, Geophys. Res. Lett., 35, L21101, doi:10.1029/2008GL035625, 2008.

Zesta, E., Lyons, L. R., and Donovan, E.: The auroral signature of Earthward flow bursts observed in the Magnetotail, Geophys. Res. Lett., 27, 3241-3244, 2000. 